\title{
On-line regression competitive with reproducing kernel Hilbert spaces
}

\author{
Vladimir Vovk \\ vovk@cs.rhul.ac.uk \\ http://vovk.net
}

Novemeber 15, 2005

\begin{abstract}
We consider the problem of on-line prediction of real-valued labels of new objects. The prediction algorithm's performance is measured by the squared deviation of the predictions from the actual labels. No probabilistic assumptions are made about the way the labels and objects are generated. Instead, we are given a benchmark class of prediction rules some of which are hoped to produce good predictions. We show that for a wide range of infinite-dimensional benchmark classes one can construct a prediction algorithm whose cumulative loss over the first $N$ examples does not exceed the cumulative loss of any prediction rule in the class plus $O(\sqrt{ } N)$. Our proof technique is based on the recently developed method of defensive forecasting.
\end{abstract}

\section{Introduction}

This paper proposes a new proof technique in competitive on-line regression, i.e., in the on-line problem of prediction of real-valued labels with the goal of performing almost as well as the best rules in a given benchmark class of prediction rules. The technique has its origin in Foster and Vohra's paper [17, which demonstrated the existence of a randomized forecasting strategy that produces asymptotically well-calibrated forecasts with probability one. Foster and Vohra's result was translated into the game-theoretic foundations of probability (see, e.g., 35]) in [45. In June 2004 Akimichi Takemura further developed the method of [45] showing that for any continuous game-theoretic law of probability there exists a forecasting strategy that perfectly satisfies this law of probability; such a strategy was called a "defensive forecasting strategy" in [46. An important special case of defensive forecasting is where the law of probability asserts good calibration and resolution of the forecasts; it was explored in 44, where, in particular, a non-asymptotic version of Foster and Vohra's result was proved. In 43 it was shown that the corresponding forecasting strategies lead to a small cumulative loss in a fairly wide class of decision protocols. That 
paper only dealt with the case of binary classification, and in this paper similar results are proved for on-line regression. As the loss function we use square-loss, which leads to significant simplifications as compared with [43].

Our main results are stated in $\$ 2$ and proved in $\S ₫ 6$ (Despite [17] being the source of our approach, our proof techniques appear to have lost all connections with that paper and papers, such as [27, 32, 33, 22, further developing it.) In \$3 these results are applied to the case where the objects and their labels are drawn independently from the same distribution. In w4 we consider some interesting benchmark classes of prediction rules, and in $\$ 5$ we compare our results to some related ones in the literature. The last section, 8 gives an explicit description of our prediction algorithms.

\section{Main results}

The simple perfect-information protocol of this section is:

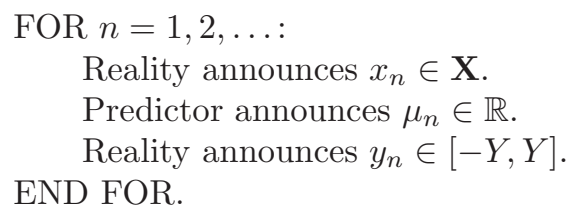

At the beginning of each round $n$ Predictor is shown an object $x_{n}$ whose label $y_{n}$ is to be predicted. The set of a priori possible objects is called the object space and denoted $\mathbf{X}$; of course, we always assume $\mathbf{X} \neq \emptyset$. After Predictor announces his prediction $\mu_{n}$ for the object's label he is shown the actual label $y_{n} \in \mathbb{R}$. We assume known an a priori upper bound $Y \in(0, \infty)$ on the absolute values of the labels $y_{n}$. We will sometimes refer to pairs $\left(x_{n}, y_{n}\right)$ as examples.

Predictor's loss on round $n$ is measured by $\left(y_{n}-\mu_{n}\right)^{2}$, and so his cumulative loss after $N$ rounds of the game is $\sum_{n=1}^{N}\left(y_{n}-\mu_{n}\right)^{2}$. His goal is "universal prediction", in the following, rather narrow, sense. If $D: \mathbf{X} \rightarrow \mathbb{R}$ is a "prediction rule" (i.e., the function $D$ is interpreted as a rule for choosing the prediction based on the current object), he would like to have

$$
\sum_{n=1}^{N}\left(y_{n}-\mu_{n}\right)^{2} \lesssim \sum_{n=1}^{N}\left(y_{n}-D\left(x_{n}\right)\right)^{2}
$$

$(\lesssim$ meaning "is not much greater than") provided $D$ is not "too complex". Technically, we will be interested in the case where the prediction rule $D$ is assumed to belong to a reproducing kernel Hilbert space (to be defined shortly) and the complexity of $D$ is measured by its norm.

\section{Reproducing kernel Hilbert spaces}

A reproducing kernel Hilbert space (RKHS) on a set $Z$ (such as $Z=\mathbf{X}$ ) is a Hilbert space $\mathcal{F}$ of real-valued functions on $Z$ such that the evaluation functional 
$f \in \mathcal{F} \mapsto f(z)$ is continuous for each $z \in Z$. We will use the notation $\mathbf{c}_{\mathcal{F}}(z)$ for the norm of this functional:

$$
\mathbf{c}_{\mathcal{F}}(z):=\sup _{f:\|f\|_{\mathcal{F}} \leq 1}|f(z)| .
$$

Let

$$
\mathbf{c}_{\mathcal{F}}:=\sup _{z \in Z} \mathbf{c}_{\mathcal{F}}(z) ;
$$

we will be interested in the case $\mathbf{c}_{\mathcal{F}}<\infty$.

Examples of RKHS will be given in

\section{Main theorems}

Suppose Predictor's goal is to compete with prediction rules $D$ from an RKHS $\mathcal{F}$ on $\mathbf{X}$. The two theorems that we state in this subsection bound the difference between the left-hand and right-hand sides of (10. The slightly simpler bound given in the first theorem is in terms of $\mathbf{c}_{\mathcal{F}},\|D\|_{\mathcal{F}}$, and $N$.

Theorem 1 Let $\mathcal{F}$ be an $R K H S$ on $\mathbf{X}$. There exists a prediction algorithm producing $\mu_{n} \in[-Y, Y]$ that are guaranteed to satisfy

$$
\sum_{n=1}^{N}\left(y_{n}-\mu_{n}\right)^{2} \leq \sum_{n=1}^{N}\left(y_{n}-D\left(x_{n}\right)\right)^{2}+2 Y \sqrt{\mathbf{c}_{\mathcal{F}}^{2}+Y^{2}}\left(\|D\|_{\mathcal{F}}+1\right) \sqrt{N}
$$

for all $N=1,2, \ldots$ and all $D \in \mathcal{F}$.

The bound in the second theorem is in terms of $\mathbf{c}_{\mathcal{F}},\|D\|_{\mathcal{F}}$, and the cumulative loss of $D$ (which can be significantly less than $N$ ).

Theorem 2 Let $\mathcal{F}$ be an $R K H S$ on $\mathbf{X}$. There exists a prediction algorithm producing $\mu_{n} \in[-Y, Y]$ that are guaranteed to satisfy

$$
\begin{aligned}
& \sum_{n=1}^{N}\left(y_{n}-\mu_{n}\right)^{2} \leq \sum_{n=1}^{N}\left(y_{n}-D\left(x_{n}\right)\right)^{2} \\
&+2 \sqrt{\mathbf{c}_{\mathcal{F}}^{2}+Y^{2}}\left(\|D\|_{\mathcal{F}}+1\right) \sqrt{\sum_{n=1}^{N}\left(y_{n}-D\left(x_{n}\right)\right)^{2}+\left(\mathbf{c}_{\mathcal{F}}^{2}+Y^{2}\right)\left(\|D\|_{\mathcal{F}}+1\right)^{2}} \\
&+\left(\mathbf{c}_{\mathcal{F}}^{2}+Y^{2}\right)\left(\|D\|_{\mathcal{F}}+1\right)^{2}
\end{aligned}
$$

for all $N$ and all $D \in \mathcal{F}$.

The bound of Theorem 2 is close to being stronger than that of Theorem 1 the former is at most twice as large as the latter plus an additive constant, if we restrict our attention to the prediction rules $D$ such that $\|D\|_{\mathcal{F}}$ is bounded by a constant and $|D(x)| \leq Y, \forall x \in \mathbf{X}$.

Prediction algorithms achieving (3) and (4) will be stated explicitly in 8 


\section{Implications for statistical learning theory}

So far we have not made any stochastic assumptions about the way the examples are produced. In this section we derive simple corollaries from Theorem 1 for the statistical learning framework, assuming that the examples $\left(x_{n}, y_{n}\right)$ are drawn independently from some probability distribution on $\mathbf{X} \times[-Y, Y]$.

The risk of a prediction rule $D: \mathbf{X} \rightarrow \mathbb{R}$ with respect to a probability distribution $P$ on $\mathbf{X} \times[-Y, Y]$ is defined as

$$
\operatorname{risk}_{P}(D):=\int(y-D(x))^{2} P(\mathrm{~d} x, \mathrm{~d} y) .
$$

(In Corollaries 1and2 we only consider prediction rules taking values in $[-Y, Y]$; this is not a real restriction if the RKHS $\mathcal{F}$ satisfies $D \in \mathcal{F} \Longrightarrow|D| \in \mathcal{F}$, as the examples of RKHS considered in 4 do.) Our goal in this section is to construct, from a given sample, a prediction rule whose risk is competitive with the risk of small-norm prediction rules in a given RKHS. As shown in 9] (with similar results obtained earlier in [7] and before that in [28), this can be easily done once we have a competitive on-line algorithm (such as those in Theorems 1 and (2).

Fix an on-line prediction algorithm (i.e., Predictor's strategy in the protocol given at the beginning of $2_{2}$ and a sequence of examples $\left(x_{1}, y_{1}\right),\left(x_{2}, y_{2}\right), \ldots$. For each $n=1,2, \ldots$, let $H_{n}: \mathbf{X} \rightarrow \mathbb{R}$ be the function that maps each $x \in \mathbf{X}$ to the prediction $\mu_{n} \in \mathbb{R}$ output by the algorithm when fed with $\left(x_{1}, y_{1}\right), \ldots,\left(x_{n-1}, y_{n-1}\right), x$. We will say that the prediction rule

$$
\bar{H}_{N}(x):=\frac{1}{N} \sum_{n=1}^{N} H_{n}(x)
$$

is obtained by averaging from the prediction algorithm.

Corollary 1 Let $\mathcal{F}$ be an $R K H S$ on $\mathbf{X}$, let $D \in \mathcal{F}$ be such that $D(x) \in[-Y, Y]$ for all $x \in \mathbf{X}$, and let $\bar{H}_{N}, N=1,2, \ldots$, be the prediction rule obtained by averaging from some prediction algorithm guaranteeing [3). For any probability distribution $P$ on $\mathbf{X} \times[-Y, Y]$, any $N=1,2, \ldots$, and any $\delta>0$,

$$
\operatorname{risk}_{P}\left(\bar{H}_{N}\right) \leq \operatorname{risk}_{P}(D)+\frac{2 Y}{\sqrt{N}}\left(\sqrt{\mathbf{c}_{\mathcal{F}}^{2}+Y^{2}}\left(\|D\|_{\mathcal{F}}+1\right)+2 Y \sqrt{2 \ln \frac{2}{\delta}}\right)
$$

with probability at least $1-\delta$.

Proof Let $\epsilon>0$. Outside an event of probability

$$
\delta:=2 \exp \left(-\frac{\epsilon^{2} N}{8 Y^{4}}\right)
$$


we have (some steps will be explained later on)

$$
\begin{aligned}
\operatorname{risk}_{P}\left(\bar{H}_{N}\right) & \leq \frac{1}{N} \sum_{n=1}^{N} \operatorname{risk}_{P}\left(H_{n}\right) \\
& \leq \frac{1}{N} \sum_{n=1}^{N}\left(y_{n}-H_{n}\left(x_{n}\right)\right)^{2}+\epsilon \\
& \leq \frac{1}{N} \sum_{n=1}^{N}\left(y_{n}-D\left(x_{n}\right)\right)^{2}+\frac{2 Y}{\sqrt{N}} \sqrt{\mathbf{c}_{\mathcal{F}}^{2}+Y^{2}}\left(\|D\|_{\mathcal{F}}+1\right)+\epsilon \\
& \leq \frac{1}{N} \sum_{n=1}^{N} \operatorname{risk}_{P}(D)+\frac{2 Y}{\sqrt{N}} \sqrt{\mathbf{c}_{\mathcal{F}}^{2}+Y^{2}}\left(\|D\|_{\mathcal{F}}+1\right)+2 \epsilon \\
& =\operatorname{risk}_{P}(D)+\frac{2 Y}{\sqrt{N}} \sqrt{\mathbf{c}_{\mathcal{F}}^{2}+Y^{2}}\left(\|D\|_{\mathcal{F}}+1\right)+2 \epsilon .
\end{aligned}
$$

The first inequality, (7), follows from the convexity of the function $t \mapsto t^{2}$. Inequalities (8) and (10) follow from Hoeffding's martingale inequality (21], Theorem 1 and the remark at the end of $\S 2$; see also 13, Theorem 9.1 on p. 135). Either of (8) and (10) holds with probability at least $1-\delta / 2$; therefore, both will hold with probability at least $1-\delta$. Finally, inequality (9) follows from Theorem [1

Our goal, (5), follows from the inequality between the extreme terms of (7)-(11) if we substitute

$$
\epsilon=\frac{2 Y^{2}}{\sqrt{N}} \sqrt{2 \ln \frac{2}{\delta}}
$$

(which is a different way of writing (6) ).

Corollary 1 can be restated using Valiant's notion of sample complexity (see, e.g., [13, $\$ 12.6$, p. 201). Let us say that the sample complexity of a class $\mathcal{C}$ of prediction rules does not exceed $N$ if it is possible to produce a prediction rule $H$ satisfying

$$
\operatorname{risk}_{P}(H) \leq \inf _{D \in \mathcal{C}} \operatorname{risk}_{P}(D)+\epsilon
$$

with probability at least $1-\delta$ when given $N$ examples generated independently from the same probability distribution $P$. The sample complexity of $\mathcal{C}$ can now be defined as the smallest $N$ with this property.

Corollary 2 For every $R>0$, the sample complexity of the restricted $R$-ball

$$
\left\{D \in \mathcal{F} \mid\|D\|_{\mathcal{F}} \leq R \& D(\mathbf{X}) \subseteq[-Y, Y]\right\}
$$

in $\mathcal{F}$ does not exceed

$$
\frac{8 Y^{2}}{\epsilon^{2}}\left(\left(\mathbf{c}_{\mathcal{F}}^{2}+Y^{2}\right)(R+1)^{2}+8 Y^{2} \ln \frac{2}{\delta}\right)+1 .
$$


Proof According to [5] we can take

$$
\epsilon=\frac{2 Y}{\sqrt{N}}\left(\sqrt{\mathbf{c}_{\mathcal{F}}^{2}+Y^{2}}(R+1)+2 Y \sqrt{2 \ln \frac{2}{\delta}}\right) .
$$

Expressing $\sqrt{ } N$ through $\epsilon$, we obtain

$$
\begin{aligned}
N \leq\left(\frac { 2 Y } { \epsilon } \left(\sqrt{\mathbf{c}_{\mathcal{F}}^{2}+Y^{2}}(R+1)+\right.\right. & \left.\left.2 Y \sqrt{2 \ln \frac{2}{\delta}}\right)\right)^{2} \\
& \leq \frac{8 Y^{2}}{\epsilon^{2}}\left(\left(\mathbf{c}_{\mathcal{F}}^{2}+Y^{2}\right)(R+1)^{2}+8 Y^{2} \ln \frac{2}{\delta}\right)
\end{aligned}
$$

(we have used the inequality $(a+b)^{2} \leq 2 a^{2}+2 b^{2}$ ). The " +1 " in (14) takes care of the possibility that the inf in (13) may not be attained.

The next corollary is a "half-way" version of Corollary 1 but it has an independent interest.

Corollary 3 Let $\mathcal{F}$ be an RKHS on $\mathbf{X}$, and let $\bar{H}_{N}, N=1,2, \ldots$, be the prediction rule obtained by averaging from some prediction algorithm guaranteeing (3). For any probability distribution $P$ on $\mathbf{X} \times[-Y, Y]$, any $N=1,2, \ldots$, and any $\delta>0$, the inequality

$$
\begin{aligned}
\operatorname{risk}_{P}\left(\bar{H}_{N}\right) \leq \frac{1}{N} \sum_{n=1}^{N}\left(y_{n}\right. & \left.-D\left(x_{n}\right)\right)^{2} \\
& +\frac{2 Y}{\sqrt{N}}\left(\sqrt{\mathbf{c}_{\mathcal{F}}^{2}+Y^{2}}\left(\|D\|_{\mathcal{F}}+1\right)+Y \sqrt{2 \ln \frac{1}{\delta}}\right)
\end{aligned}
$$

holds with probability at least $1-\delta$ for all $D \in \mathcal{F}$ simultaneously.

Proof Outside an event whose probability is at most half of (6) we have (7)-(9); it remains to set

$$
\epsilon=\frac{2 Y^{2}}{\sqrt{N}} \sqrt{2 \ln \frac{1}{\delta}}
$$

instead of (12).

Since (15) holds with probability at least $1-\delta$ for all $D$ simultaneously, it can be applied to a $D$ chosen a posteriori (say, by the regularized least squares); we will still be able to say that 15) holds unless a rare event (of probability at most $\delta$ ) occurred. In fact, Corollary 3 can be equivalently restated to say that

$$
\begin{aligned}
\operatorname{risk}_{P}\left(\bar{H}_{N}\right) \leq \inf _{D \in \mathcal{F}}\left(\frac{1}{N} \sum_{n=1}^{N}\right. & \left(y_{n}-D\left(x_{n}\right)\right)^{2} \\
& \left.+\frac{2 Y}{\sqrt{N}}\left(\sqrt{\mathbf{c}_{\mathcal{F}}^{2}+Y^{2}}\left(\|D\|_{\mathcal{F}}+1\right)+Y \sqrt{2 \ln \frac{1}{\delta}}\right)\right)
\end{aligned}
$$

holds with probability at least $1-\delta$. 


\section{Examples of RKHS and reproducing kernels}

The usefulness of the theorems stated in the previous two sections depends on the availability of suitable RKHS. For numerous examples of useful RKHS see, e.g., 41, 34, and 36]; in this section I will only give three simple examples of RKHS.

\section{The Sobolev spaces}

The Sobolev norm $\|f\|_{H^{1}}$ of a smooth function $f:[0,1] \rightarrow \mathbb{R}$ is defined by

$$
\|f\|_{H^{1}}^{2}:=\int_{0}^{1}(f(t))^{2} \mathrm{~d} t+\int_{0}^{1}\left(f^{\prime}(t)\right)^{2} \mathrm{~d} t .
$$

The Sobolev space $H^{1}([0,1])$ on $[0,1]$ is the completion of the set of smooth $f:[0,1] \rightarrow \mathbb{R}$ satisfying $\|f\|_{H^{1}}<\infty$ with respect to the norm $\|\cdot\|_{H^{1}}$. It is easy to see that $H^{1}([0,1])$ is an RKHS. In fact, it is only one of a range of Sobolev spaces (see, e.g., [1] for the definition of the full range; the space $H^{1}([0,1])$ is the "least smooth" among Sobolev spaces $H^{s}([0,1])$ on $[0,1]$ if we ignore the slightly less natural case of a fractional $s$ ).

An important advantage of the Sobolev class $H^{1}([0,1])$ over some other classes used in machine learning is that it is universal, in the sense that it is dense in the set of all continuous functions on [0,1] (cf. [39], Definition 4).

It is easy to see that neither of the two addends in (16) can be omitted: if the first addend is omitted, the square root of the right-hand side of (16) ceases to be a norm (since it becomes zero for every constant), and if the second addend is omitted, it ceases to be an RKHS (since the evaluation functionals become unbounded). We can, however, "partially omit" the first addend replacing (16) with the Fermi-Sobolev norm $\|f\|_{\mathrm{FS}}$ defined by

$$
\|f\|_{\mathrm{FS}}^{2}:=\left(\int_{0}^{1} f(t) \mathrm{d} t\right)^{2}+\int_{0}^{1}\left(f^{\prime}(t)\right)^{2} \mathrm{~d} t
$$

for smooth functions $f:[0,1] \rightarrow \mathbb{R}$. The Fermi-Sobolev space on $[0,1]$ is the completion of the set of smooth $f:[0,1] \rightarrow \mathbb{R}$ satisfying $\|f\|_{\mathrm{FS}}<\infty$ with respect to the norm $\|\cdot\|_{\mathrm{FS}}$. It is clear that it is still an RKHS, and it is still universal.

Remark The Fermi-Sobolev space is very popular in statistics: see, e.g., 47, pp. 129-130, or [19], §2.3.3. Surprisingly, it does not have a name; in this draft I use the name I gave to it in the early versions of [44, before learning how popular it was.

Of course, the underlying set $Z$ of an RKHS does not have to be a compact topological space: we can define the Sobolev norm $\|f\|_{H^{1}}$ of a smooth function $f: \mathbb{R} \rightarrow \mathbb{R}$ by essentially the same formula

$$
\|f\|_{H^{1}}^{2}:=\int_{-\infty}^{\infty}(f(t))^{2} \mathrm{~d} t+\int_{-\infty}^{\infty}\left(f^{\prime}(t)\right)^{2} \mathrm{~d} t
$$


and define the Sobolev space $H^{1}(\mathbb{R})$ on $\mathbb{R}$ as the completion of the set of smooth $f: \mathbb{R} \rightarrow \mathbb{R}$ satisfying $\|f\|_{H^{1}}<\infty$.

To apply Theorems 1 and 2 to these RKHS we need to know the value of $\mathbf{c}_{\mathcal{F}}$ for them; later in this section we will see that

$$
\mathbf{c}_{\mathcal{F}}=\mathbf{c}_{H^{1}([0,1])}=\sqrt{\operatorname{coth} 1} \approx 1.15
$$

for the Sobolev space $H^{1}([0,1])$,

$$
\mathbf{c}_{\mathcal{F}}=\mathbf{c}_{\mathrm{FS}}=2 / \sqrt{3} \approx 1.15
$$

for the Fermi-Sobolev space on $[0,1]$, and

$$
\mathbf{c}_{\mathcal{F}}=\mathbf{c}_{H^{1}(\mathbb{R})}=1 / \sqrt{2} \approx 0.71
$$

for the Sobolev space $H^{1}(\mathbb{R})$.

Remark The term "Sobolev space" usually serves as the name for a topological vector space; all these spaces are normable, but different norms are not considered to lead to different Sobolev spaces as long as the topology does not change. The norms given by (16) and (18) are the most standard ones. It is easy to see that the norm (17) leads to the same topology as (16): $\|f\|_{\mathrm{FS}} \leq\|f\|_{H^{1}}$ is obvious, and $\|f\|_{H^{1}}=O\left(\|f\|_{\mathrm{FS}}\right)$ follows from Wirtinger's inequality (which implies that $\int_{0}^{\pi} f^{2} \leq \int_{0}^{\pi}\left(f^{\prime}\right)^{2}$ for every function $f$ on $[0, \pi]$ such that $f^{\prime}$ is in $L_{2}$ and $\int_{0}^{\pi} f=0$; for the statement and a proof of Wirtinger's inequality, see [20, Theorem 258).

Remark We glossed over one important point in our discussion: in principle, the completion of a pre-Hilbert space with bounded evaluation functionals is not guaranteed to be an RKHS. If no extra precautions are taken, the usual procedure of completion even destroys the structure of a function space (the ideal elements added with each equivalence class of Cauchy sequences are not necessarily functions). However, the theorem in $\S$ I.4 of 3] guarantees that in each of our three examples there is a unique "functional completion", which is an RKHS (that theorem, however, does involve a non-trivial condition on the original incomplete space). These functional completions can be described explicitly: the Sobolev space $H^{1}([0,1])\left(\right.$ resp. $\left.H^{1}(\mathbb{R})\right)$ consists of all absolutely continuous functions on $[0,1]$ (resp. on $\mathbb{R}$ ) for which $\|f\|_{H^{1}}$ is finite, and similarly, the Fermi-Sobolev space on $[0,1]$ consists of all absolutely continuous functions on $[0,1]$ for which $\|f\|_{\mathrm{FS}}$ is finite (for the Sobolev spaces this follows from [6], Theorem 129 on p. 348, and [1, Theorem 3.22 on p. 68, and for the Fermi-Sobolev space this can then be deduced using the previous remark; for an independent derivation for the Fermi-Sobolev space, see [4], version 2, Lemmas 9 and 10). This explicit description provides an alternative, constructive, proof of the existence of a functional completion; the uniqueness is asserted by the theorem in $\S \mathrm{I} .4$ of [3]. 
We are often interested in the case where the objects $x_{n}$ are vectors in a Euclidean space $\mathbb{R}^{m}$; if their components are bounded, we can scale them so that $x_{n} \in[0,1]^{m}$ and take the $m$ th tensor power $\mathcal{F}$ of one of the three RKHS we have just defined as our benchmark class. (For the definition and properties of tensor products of RKHS see, e.g., [3], §I.8.) We will see later that $\mathbf{c}_{\mathcal{F}}$ for the $m$ th tensor power is the $m$ th power of the $\mathbf{c}_{\mathcal{F}}$ for the original RKHS. The $m$ th tensor power of the Sobolev and Fermi-Sobolev spaces on $[0,1]$ are universal on $[0,1]^{m}$, in the sense of being dense in the set of all continuous functions on $[0,1]^{m}$ (this can be seen from the construction given in [3], $\S$ I.8).

\section{Reproducing kernels}

An equivalent language for talking about RKHS is provided by the notion of a reproducing kernel; this subsection defines reproducing kernels and summarizes some of their properties. For a detailed discussion, see, e.g., 2]-3] or [30].

Let $\mathcal{F}$ be an RKHS on $Z$. By the Riesz-Fischer theorem, for each $z \in Z$ there exists a function $\mathbf{k}_{z} \in \mathcal{F}$ such that

$$
f(z)=\left\langle\mathbf{k}_{z}, f\right\rangle_{\mathcal{F}}, \quad \forall f \in \mathcal{F}
$$

The next lemma asserts that $\left\|\mathbf{k}_{z}\right\|_{\mathcal{F}}$ is the norm $\mathbf{c}_{\mathcal{F}}(z)$ of the evaluation functional $f \mapsto f(z)$.

Lemma 1 Let $\mathcal{F}$ be an $R K H S$ on $Z$. For each $z \in Z$,

$$
\left\|\mathbf{k}_{z}\right\|_{\mathcal{F}}=\mathbf{c}_{\mathcal{F}}(z)
$$

Proof Fix $z \in Z$. We are required to prove

$$
\sup _{f:\|f\|_{\mathcal{F}} \leq 1}|f(z)|=\left\|\mathbf{k}_{z}\right\|_{\mathcal{F}}
$$

The inequality $\leq$ follows from

$$
|f(z)|=\left|\left\langle f, \mathbf{k}_{z}\right\rangle_{\mathcal{F}}\right| \leq\|f\|_{\mathcal{F}}\left\|\mathbf{k}_{z}\right\|_{\mathcal{F}} \leq\left\|\mathbf{k}_{z}\right\|_{\mathcal{F}}
$$

where $\|f\|_{\mathcal{F}} \leq 1$. The inequality $\geq$ follows from

$$
|f(z)|=\frac{\mathbf{k}_{z}(z)}{\left\|\mathbf{k}_{z}\right\|_{\mathcal{F}}}=\frac{\left\langle\mathbf{k}_{z}, \mathbf{k}_{z}\right\rangle_{\mathcal{F}}}{\left\|\mathbf{k}_{z}\right\|_{\mathcal{F}}}=\left\|\mathbf{k}_{z}\right\|_{\mathcal{F}}
$$

where $f:=\mathbf{k}_{z} /\left\|\mathbf{k}_{z}\right\|_{\mathcal{F}}$.

The reproducing kernel of $\mathcal{F}$ is the function $\mathbf{k}: Z^{2} \rightarrow \mathbb{R}$ defined by

$$
\mathbf{k}\left(z, z^{\prime}\right):=\left\langle\mathbf{k}_{z}, \mathbf{k}_{z^{\prime}}\right\rangle_{\mathcal{F}}
$$

(equivalently, we could define $\mathbf{k}\left(z, z^{\prime}\right)$ as $\mathbf{k}_{z}\left(z^{\prime}\right)$ or as $\mathbf{k}_{z^{\prime}}(z)$ ). The origin of this name is the "reproducing property" (19). 
There is a simple internal characterization of reproducing kernels of RKHS. First, it is easy to check that the function $\mathbf{k}\left(z, z^{\prime}\right)$, as we defined it, is symmetric,

$$
\mathbf{k}\left(z, z^{\prime}\right)=\mathbf{k}\left(z^{\prime}, z\right), \quad \forall\left(z, z^{\prime}\right) \in Z^{2},
$$

and positive definite,

$$
\begin{aligned}
& \sum_{i=1}^{m} \sum_{j=1}^{m} \alpha_{i} \alpha_{j} \mathbf{k}\left(z_{i}, z_{j}\right) \geq 0, \\
& \forall m=1,2, \ldots,\left(\alpha_{1}, \ldots, \alpha_{m}\right) \in \mathbb{R}^{m},\left(z_{1}, \ldots, z_{m}\right) \in Z^{m} .
\end{aligned}
$$

On the other hand, for every symmetric and positive definite $\mathbf{k}: Z^{2} \rightarrow \mathbb{R}$ there exists a unique RKHS $\mathcal{F}$ such that $\mathbf{k}$ is the reproducing kernel of $\mathcal{F}$ ([2], Theorem 2 on p. 143).

We can see that the notions of a reproducing kernel of RKHS and of a symmetric positive definite function on $Z^{2}$ have the same content, and we will sometimes say "kernel on $Z$ " to mean a symmetric positive definite function on $Z^{2}$. Kernels in this sense are the main source of RKHS in learning theory; see, e.g., 41], 34, and [36] for numerous examples. Every kernel on $\mathbf{X}$ is a valid parameter for our prediction algorithms; to apply Theorems 1 and 2 we can use the equivalent definition of $\mathbf{c}_{\mathcal{F}}$,

$$
\mathbf{c}_{\mathcal{F}}=\mathbf{c}_{\mathbf{k}}:=\sup _{x \in \mathbf{X}} \sqrt{\mathbf{k}(x, x)},
$$

$\mathrm{k}$ being the reproducing kernel of $\mathcal{F}$.

It was convenient to start from RKHS in stating the theorems of $\$ 2$ but our prediction algorithms, explicitly described in $\$ 8$ will use the more constructive representation of RKHS via their reproducing kernels.

\section{Norm vs. the reproducing kernel in RKHS}

Finding the norm given the reproducing kernel and vice versa are often nontrivial problems for specific RKHS. The most popular methods appear to be the following.

- As we saw in the proof of Lemma $1 \mathbf{k}_{z} /\left\|\mathbf{k}_{z}\right\|_{\mathcal{F}}$ is the function at which

$$
\sup _{f:\|f\|_{\mathcal{F}} \leq 1}|f(z)|
$$

is attained (assuming this optimization problem has a unique solution). Solving this optimization problem we can find the kernel $\mathbf{k}$ given the norm $f \mapsto\|f\|_{\mathcal{F}}$. For application of this method to the Fermi-Sobolev space on $[0,1]$, see [44], Appendix C.

- One can use expansions into Fourier series of functions in a given RKHS. For examples see, e.g., 19, §4.2.1, or, for the Fermi-Sobolev space on $[0,1]$, 44] (version 2). 
- If $Z$ is a Euclidean space and the reproducing kernel $\mathbf{k}\left(z, z^{\prime}\right)$ only depends on the difference $z-z^{\prime}$ (is "translation-invariant"), an explicit formula for the reproducing kernel can sometimes be obtained by applying the Fourier transform to both sides of (19) (similar methods are applied to the Sobolev space $H^{1}(\mathbb{R})$ in $[40$ and $[38$ ).

The reproducing kernel of the Sobolev space $H^{1}([0,1])$, as given in [6] (\$7.4, Example 13; Exercise 3.12.7) with a reference to [4, is

$$
\mathbf{k}\left(t, t^{\prime}\right)=\frac{\cosh \min \left(t, t^{\prime}\right) \cosh \min \left(1-t, 1-t^{\prime}\right)}{\sinh 1} .
$$

It is easy to find that

$$
\mathbf{c}_{\mathbf{k}}^{2}=\sup _{t \in[0,1]} \frac{\cosh t \cosh (1-t)}{\sinh 1}=\frac{\cosh 0 \cosh 1}{\sinh 1}=\operatorname{coth} 1,
$$

as stated above.

The reproducing kernel of the Fermi-Sobolev space on $[0,1]$ was found in 11] (see also [47], $\S 10.2$, or [19], $\S 2.3 .3$ ); it is given by

$$
\begin{aligned}
\mathbf{k}\left(t, t^{\prime}\right) & =k_{0}(t) k_{0}\left(t^{\prime}\right)+k_{1}(t) k_{1}\left(t^{\prime}\right)+k_{2}\left(\left|t-t^{\prime}\right|\right) \\
& =1+\left(t-\frac{1}{2}\right)\left(t^{\prime}-\frac{1}{2}\right)+\frac{1}{2}\left(\left|t-t^{\prime}\right|^{2}-\left|t-t^{\prime}\right|+\frac{1}{6}\right) \\
& =\frac{1}{2} \min ^{2}\left(t, t^{\prime}\right)+\frac{1}{2} \min ^{2}\left(1-t, 1-t^{\prime}\right)+\frac{5}{6},
\end{aligned}
$$

where $k_{l}:=B_{l} / l$ ! are scaled Bernoulli polynomials $B_{l}$. So, for the Fermi-Sobolev space on $[0,1]$ we have

$$
\mathbf{c}_{\mathbf{k}}^{2}=\max _{t \in[0,1]}\left(\frac{1}{2} t^{2}+\frac{1}{2}(1-t)^{2}+\frac{5}{6}\right)=\frac{4}{3} .
$$

The reproducing kernel of the Sobolev space $H^{1}(\mathbb{R})$ is

$$
\mathbf{k}\left(t, t^{\prime}\right)=\frac{1}{2} \exp \left(-\left|t-t^{\prime}\right|\right)
$$

(see [4], 38, or [6], §7.4, Example 24). From the last equation we can see that $\mathbf{c}_{H^{1}(\mathbb{R})}=1 / \sqrt{2}$.

It is the general fact that the reproducing kernel of the $m$-fold product of RKHS can be obtained as the $m$-fold product of the reproducing kernels of the components ([3], §I.8, Theorem I). For example, the reproducing kernel of the $m$ th power of $H^{1}([0,1])$ is

$$
\mathbf{k}\left(\left(t_{1}, \ldots, t_{m}\right),\left(t_{1}^{\prime}, \ldots, t_{m}^{\prime}\right)\right)=\prod_{i=1}^{m} \frac{\cosh \min \left(t_{i}, t_{i}^{\prime}\right) \cosh \min \left(1-t_{i}, 1-t_{i}^{\prime}\right)}{\sinh 1} .
$$


We can see that

$$
\mathbf{c}_{\mathcal{F}}=(\operatorname{coth} 1)^{m / 2}, \quad \mathbf{c}_{\mathcal{F}}=(2 / \sqrt{3})^{m}, \quad \mathbf{c}_{\mathcal{F}}=2^{-m / 2}
$$

for the $m$ th power of the Sobolev space $H^{1}([0,1])$, of the Fermi-Sobolev space on $[0,1]$, and of the Sobolev space $H^{1}(\mathbb{R})$, respectively.

An extensive list of RKHS together with their reproducing kernels is given in [6], $\$ 7.4$.

\section{Some comparisons}

In this section I will state several results from literature related to Theorems

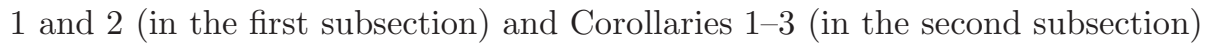
that I happen to know about. This part of the paper is especially sketchy.

\section{Competitive on-line learning}

The first paper about competitive on-line regression is [16], and [10] and [25] are important milestones; for a brief review (written for statisticians) of the work done in the 1990s see 42. This is a typical result: if the benchmark class $\mathcal{F}$ consists of the linear functions $D(x)=\langle\theta, x\rangle$ on $\mathbf{X}=\mathbb{R}^{m}$ whose "complexity" is measured by the $L_{2}$ norm $\|\theta\|_{2}:=\sqrt{\sum_{i=1}^{m} \theta_{i}^{2}}$ of $\theta$ 's components $\theta_{i}$ and if $a$ is a positive constant, some prediction algorithm ensures

$$
\begin{aligned}
& \sum_{n=1}^{N}\left(y_{n}-\mu_{n}\right)^{2} \\
& \leq \sum_{n=1}^{N}\left(y_{n}-\left\langle\theta, x_{n}\right\rangle\right)^{2}+a\|\theta\|_{2}^{2}+Y^{2} \ln \operatorname{det}\left(I+\frac{1}{a} \sum_{n=1}^{N} x_{n} x_{n}^{\prime}\right) \\
& \leq \sum_{n=1}^{N}\left(y_{n}-\left\langle\theta, x_{n}\right\rangle\right)^{2}+a\|\theta\|_{2}^{2}+Y^{2} \sum_{i=1}^{m} \ln \left(1+\frac{1}{a} \sum_{n=1}^{N} x_{n, i}^{2}\right),
\end{aligned}
$$

for all $N$ and all $\theta \in \mathbb{R}^{m}$ (42], Theorem 1; different proofs are given in [5], Theorem 4.6, and [15]). In particular, if $\|\theta\|_{2}$ and all components $x_{n, i}$ of all $x_{n}$ are bounded by a constant,

$$
\sum_{n=1}^{N}\left(y_{n}-\mu_{n}\right)^{2} \leq \sum_{n=1}^{N}\left(y_{n}-\left\langle\theta, x_{n}\right\rangle\right)^{2}+O(\ln N) ;
$$

it is interesting that the "regret" term is now $O(\ln N)$, rather than $O(\sqrt{ } N)$ as in (3).

In this brief review we, however, concentrate on the infinite-dimensional benchmark classes. The result (21) was carried over to RKHS in [18]: there is 
a prediction algorithm that ensures

$$
\sum_{n=1}^{N}\left(y_{n}-\mu_{n}\right)^{2} \leq \sum_{n=1}^{N}\left(y_{n}-D\left(x_{n}\right)\right)^{2}+a\|D\|_{\mathcal{F}}^{2}+Y^{2} \ln \operatorname{det}\left(I+\frac{1}{a} K\right)
$$

for all $N$ and all prediction rules $D$ in an RKHS $\mathcal{F}$, where $K$ is the $N \times N$ matrix (sometimes called the "Gram matrix") with the elements $K_{i, j}:=\mathbf{k}\left(x_{i}, x_{j}\right)$, $i, j=1, \ldots, N$, and $\mathbf{k}$ is $\mathcal{F}$ 's reproducing kernel.

A disadvantage of the bound (22) is that it is not immediately clear whether the term

$$
\ln \operatorname{det}\left(I+\frac{1}{a} K\right)
$$

is small as compared with $N$. The same term occurs in 23] (Theorems 3.1 and 3.2 ) and [8].

A very interesting early paper about on-line regression competitive with function spaces is 24] (continued by 29]); it, however, assumes that the benchmark class contains a perfect prediction rule and its results are very different from ours.

\section{Statistical learning theory}

In this subsection we will review results related to our Corollaries [1] Some of these results depend on extra assumptions about the RKHS considered, but these assumptions are relatively mild and satisfied in most examples considered in the previous section. We will use the same notation that we used in Corollaries 113

Cucker and Smale's paper 12 reports a result closest to ours. Let $\mathbf{X}$ be a compact domain or manifold in $\mathbb{R}^{m}$ and $H^{s}(\mathbf{X}), s>m / 2$, be the Sobolev space on it (for the definition in the case $\mathbf{X}=[0,1]$ and $s=1$, see the previous section). Cucker and Smale ([12, Example 3 (continued) on p. 18) obtain the upper bound

$$
\frac{1152 Y^{2}}{\epsilon}\left(\left(\frac{48 C R Y}{\epsilon}\right)^{m / s}+\ln \frac{1}{\delta}\right)
$$

where $C$ is a constant (which may, however, depend on $s$ ), on the sample complexity of the restricted $R$-ball in $H^{s}(\mathbf{X})$. Therefore, for the space $H^{1}([0,1])$ we have two bounds on the sample complexity:

$$
\begin{array}{ll}
\frac{8 Y^{2}}{\epsilon^{2}}\left(\left(\operatorname{coth} 1+Y^{2}\right)(R+1)^{2}+8 Y^{2} \ln \frac{2}{\delta}\right)+1 & \text { Corollary } 2 \\
\frac{1152 Y^{2}}{\epsilon}\left(\frac{48 C R Y}{\epsilon}+\ln \frac{1}{\delta}\right) & \text { 23) with } s=1 .
\end{array}
$$

Both bounds scale as $\epsilon^{-2}$ for small $\epsilon>0$. Neither is strictly better than the other: e.g., an advantage of our bound is that all constants are given explicitly and are rather small, and an advantage of Cucker and Smale's bound is that the 
coefficient in front of $\ln (1 / \delta)$ scales as $\epsilon^{-1}$. Going outside $H^{1}([0,1])$, we have a similar bound, (14), for general RKHS (e.g., we do not need the compactness of $\mathbf{X}$ for Sobolev spaces); on the other hand, Cucker and Smale's bounds scale better in $\epsilon$ for classes of smooth functions (cf. 23) for $s>1$ and, especially, the result of [12, Example 4 (continued) on p. 18, for $C^{\infty}$ kernels; it should be noted, however, that our bound scales better in $\epsilon$ for the least smooth of the Sobolev classes, namely, for $H^{s}$ with $\left.s \in(1 / 2,1)\right)$.

Cucker and Smale's methods are based on using entropy numbers of compact operators (introduced into learning theory by [48]). A very different method, based on stability of learning algorithms, is proposed by Bousquet and Elisseeff, who prove the bound

$$
\operatorname{risk}_{P}\left(H_{N}\right) \leq \frac{1}{N} \sum_{n=1}^{N}\left(y_{n}-H_{N}\left(x_{n}\right)\right)^{2}+\frac{16 Y^{2} \mathbf{c}_{\mathcal{F}}^{2}}{\lambda N}+\left(\frac{32 Y^{2} \mathbf{c}_{\mathcal{F}}^{2}}{\lambda}+4 Y\right) \sqrt{\frac{\ln \frac{1}{\delta}}{2 N}}
$$

for the prediction rule $H_{N}=H$ obtained by the regularized least squares,

$$
\frac{1}{N} \sum_{n=1}^{N}\left(y_{n}-H\left(x_{n}\right)\right)^{2}+\lambda\|H\|_{\mathcal{F}}^{2} \rightarrow \min ,
$$

where $\lambda$ is a positive constant ([14, Example 3 in $\S 5.2 .2$, p. 517). Therefore, we have, with probability $1-\delta$ for all $D$ simultaneously,

$$
\begin{aligned}
\operatorname{risk}_{P}\left(H_{N}\right) \leq \frac{1}{N} \sum_{n=1}^{N} & \left(y_{n}-D\left(x_{n}\right)\right)^{2} \\
& +\lambda\|D\|_{\mathcal{F}}^{2}+\frac{16 Y^{2} \mathbf{c}_{\mathcal{F}}^{2}}{\lambda N}+\left(\frac{32 Y^{2} \mathbf{c}_{\mathcal{F}}^{2}}{\lambda}+4 Y\right) \sqrt{\frac{\ln \frac{1}{\delta}}{2 N}} .
\end{aligned}
$$

This is similar to our (15), except that the part following the first " + " in (24) does not tend to 0 for a fixed $\lambda$. Taking $\lambda=\lambda_{N} \rightarrow 0$ makes the dependence on $N$ in (24) worse than in (15).

There are several other results of this kind in literature, such as

$$
\mathbb{E} \operatorname{risk}_{P}\left(H_{N}\right) \leq\left(1+\frac{2 \mathbf{c}_{\mathcal{F}}^{2}}{\lambda N}\right)^{2}\left(\operatorname{risk}_{P}(D)+\frac{\lambda}{2}\|D\|_{\mathcal{F}}^{2}\right)
$$

(49, as stated in [37, (7.17)); this should be compared to Corollary 1 Some further information can be found in 31.

The traditional approach to regression in statistical learning theory [41] is based on modifications of the VC dimension, such as pseudo-dimension: e.g., Lee, Bartlett, and Williamson [26] prove that the sample complexity of a convex function class of finite pseudo-dimension is

$$
O\left(\frac{1}{\epsilon}\left(\ln \frac{1}{\epsilon}+\ln \frac{1}{\delta}\right)\right) .
$$

Unfortunately, the pseudo-dimension is infinite for many interesting benchmark classes (such as the examples of RKHS in 4 ). 


\section{Proof of Theorem [1}

This section is essentially a simplified (and to some degree cut-and-pasted) version of $\S \S 5-7$ of [43]. First we modify the protocol of $\$ 2$ introducing a third player, Skeptic, who is allowed to bet at the odds implied by Predictor's moves.

Forecasting Game I

Players: Reality, Predictor, Skeptic

Protocol:

FOR $n=1,2, \ldots$ :

Reality announces $x_{n} \in \mathbf{X}$.

Predictor announces $\mu_{n} \in \mathbb{R}$.

Skeptic announces $s_{n} \in \mathbb{R}$.

Reality announces $y_{n} \in[-Y, Y]$.

$\mathcal{K}_{n}:=\mathcal{K}_{n-1}+s_{n}\left(y_{n}-\mu_{n}\right)$.

END FOR.

In this protocol, the prediction $\mu_{n}$ is interpreted as the price Predictor charges for a ticket paying $y_{n} ; s_{n}$ is the number of tickets Skeptic decides to buy. (We sometimes refer to predictions interpreted this way as forecasts, although the difference between forecasts and the decision-type predictions of $\$ 2$ is not as important here as for the more general loss functions considered in [43.) The protocol describes not only the players' moves but also the changes in Skeptic's capital $\mathcal{K}_{n}$; its initial value $\mathcal{K}_{0}$ can be an arbitrary real number. Protocols of this type are studied extensively in 35.

For any continuous strategy for Skeptic there exists a strategy for Predictor that does not allow Skeptic's capital to grow, regardless of Reality's moves. To state this observation in its strongest form, we make Skeptic announce his strategy for each round before Predictor's move on that round rather than announce his full strategy at the beginning of the game. Therefore, we consider the following perfect-information game:

\section{Forecasting Game II}

Players: Reality, Predictor, Skeptic

Protocol:

FOR $n=1,2, \ldots$ :

Reality announces $x_{n} \in \mathbf{X}$.

Skeptic announces continuous $S_{n}: \mathbb{R} \rightarrow \mathbb{R}$.

Predictor announces $\mu_{n} \in \mathbb{R}$.

Reality announces $y_{n} \in[-Y, Y]$. $\mathcal{K}_{n}:=\mathcal{K}_{n-1}+S_{n}\left(\mu_{n}\right)\left(y_{n}-\mu_{n}\right)$.

END FOR.

Lemma 2 Predictor has a strategy in Forecasting Game II that ensures $\mu_{n} \in$ $[-Y, Y]$, for all $n=1,2, \ldots$, and $\mathcal{K}_{0} \geq \mathcal{K}_{1} \geq \mathcal{K}_{2} \geq \cdots$. 
Proof Predictor's goal is achieved by the following strategy:

- if the function $S_{n}$ takes value 0 on the interval $[-Y, Y]$, choose $\mu_{n} \in$ $[-Y, Y]$ such that $S_{n}\left(\mu_{n}\right)=0$

- if $S_{n}$ is always positive on $[-Y, Y]$, take $\mu_{n}:=Y$;

- if $S_{n}$ is always negative on $[-Y, Y]$, take $\mu_{n}:=-Y$.

\section{Algorithm of Large Numbers}

We say that a kernel $\mathbf{k}$ on $[-Y, Y] \times \mathbf{X}$ is forecast-continuous if the function $\mathbf{k}\left((\mu, x),\left(\mu^{\prime}, x^{\prime}\right)\right)$ is continuous in $\left(\mu, \mu^{\prime}\right) \in[-Y, Y]^{2}$, for all fixed $\left(x, x^{\prime}\right) \in \mathbf{X}^{2}$. For such a kernel the function

$$
S_{n}(\mu):=\sum_{i=1}^{n-1} \mathbf{k}\left(\left(\mu, x_{n}\right),\left(\mu_{i}, x_{i}\right)\right)\left(y_{i}-\mu_{i}\right)-\mathbf{k}\left(\left(\mu, x_{n}\right),\left(\mu, x_{n}\right)\right) \mu
$$

is continuous in $\mu \in[-Y, Y]$.

The ALGORITHM OF LARGE NUMBers (ALN)

Parameter: forecast-continuous kernel $\mathbf{k}$ on $[-Y, Y] \times \mathbf{X}$

FOR $n=1,2, \ldots$ :

Read $x_{n} \in \mathbf{X}$.

Define $S_{n}:[-Y, Y] \rightarrow \mathbb{R}$ by (25).

Output any root $\mu \in[-Y, Y]$ of $S_{n}(\mu)=0$ as $\mu_{n}$;

if there are no roots, set $\mu_{n}:=Y \operatorname{sign} S_{n}$.

Read $y_{n} \in[-Y, Y]$.

END FOR.

(Notice that $\operatorname{sign} S_{n}$ is well defined in this context.) It is well known that for each kernel on $\mathbf{k}$ on $[-Y, Y] \times \mathbf{X}$ there exists a function $\Phi:[-Y, Y] \times \mathbf{X} \rightarrow \mathcal{H}$ (a feature mapping taking values in a Hilbert space $\mathcal{H}$ ) such that

$$
\mathbf{k}(a, b)=\langle\Phi(a), \Phi(b)\rangle_{\mathcal{H}}, \forall a, b \in[-Y, Y] \times \mathbf{X} .
$$

(For example, we can take the RKHS on $[-Y, Y] \times \mathbf{X}$ with reproducing kernel $\mathbf{k}$ as $\mathcal{H}$ and take $a \mapsto \mathbf{k}_{a}$ as the feature mapping $\Phi$; there are, however, easier and more transparent constructions.) It can be shown that $\Phi(\mu, x)$ is forecastcontinuous, i.e., continuous in $\mu \in[-Y, Y]$ for each fixed $x \in \mathbf{X}$, if and only if the kernel $\mathbf{k}$ defined by (26) is forecast-continuous (see, e.g., 44, Appendix B, where $[0,1]$ should be replaced with $[-Y, Y])$.

Theorem 3 Let $\mathbf{k}$ be the kernel defined by (26) for a forecast-continuous feature mapping $\Phi:[-Y, Y] \times \mathbf{X} \rightarrow \mathcal{H}$, where $\mathcal{H}$ is a Hilbert space. The ALN with parameter $\mathbf{k}$ outputs $\mu_{n} \in[-Y, Y]$ such that

$$
\left\|\sum_{n=1}^{N}\left(y_{n}-\mu_{n}\right) \Phi\left(\mu_{n}, x_{n}\right)\right\|_{\mathcal{H}}^{2} \leq \sum_{n=1}^{N}\left(Y^{2}-\mu_{n}^{2}\right)\left\|\Phi\left(\mu_{n}, x_{n}\right)\right\|_{\mathcal{H}}^{2}
$$


always holds for all $N=1,2, \ldots$.

Proof Following the ALN, Predictor ensures that Skeptic will never increase his capital with the strategy

$$
s_{n}:=\sum_{i=1}^{n-1} \mathbf{k}\left(\left(\mu_{n}, x_{n}\right),\left(\mu_{i}, x_{i}\right)\right)\left(y_{i}-\mu_{i}\right)-\mathbf{k}\left(\left(\mu_{n}, x_{n}\right),\left(\mu_{n}, x_{n}\right)\right) \mu_{n} .
$$

Using the inequalities

$$
\left(y_{n}-\mu_{n}\right)^{2}+2 \mu_{n}\left(y_{n}-\mu_{n}\right) \leq Y^{2}-\mu_{n}^{2}
$$

and

$$
\mathbf{k}\left(\left(\mu_{n}, x_{n}\right),\left(\mu_{n}, x_{n}\right)\right) \geq 0
$$

we can see that the increase in Skeptic's capital when he follows (28) is

$$
\begin{aligned}
\mathcal{K}_{N}-\mathcal{K}_{0}= & \sum_{n=1}^{N} s_{n}\left(y_{n}-\mu_{n}\right) \\
= & \sum_{n=1}^{N} \sum_{i=1}^{n-1} \mathbf{k}\left(\left(\mu_{n}, x_{n}\right),\left(\mu_{i}, x_{i}\right)\right)\left(y_{n}-\mu_{n}\right)\left(y_{i}-\mu_{i}\right) \\
& -\sum_{n=1}^{N} \mathbf{k}\left(\left(\mu_{n}, x_{n}\right),\left(\mu_{n}, x_{n}\right)\right) \mu_{n}\left(y_{n}-\mu_{n}\right) \\
= & \frac{1}{2} \sum_{n=1}^{N} \sum_{i=1}^{N} \mathbf{k}\left(\left(\mu_{n}, x_{n}\right),\left(\mu_{i}, x_{i}\right)\right)\left(y_{n}-\mu_{n}\right)\left(y_{i}-\mu_{i}\right) \\
& -\frac{1}{2} \sum_{n=1}^{N} \mathbf{k}\left(\left(\mu_{n}, x_{n}\right),\left(\mu_{n}, x_{n}\right)\right)\left(y_{n}-\mu_{n}\right)^{2} \\
& -\sum_{n=1}^{N} \mathbf{k}\left(\left(\mu_{n}, x_{n}\right),\left(\mu_{n}, x_{n}\right)\right) \mu_{n}\left(y_{n}-\mu_{n}\right) \\
\geq & \frac{1}{2} \sum_{n=1}^{N} \sum_{i=1}^{N} \mathbf{k}\left(\left(\mu_{n}, x_{n}\right),\left(\mu_{i}, x_{i}\right)\right)\left(y_{n}-\mu_{n}\right)\left(y_{i}-\mu_{i}\right) \\
& -\frac{1}{2} \sum_{n=1}^{N} \mathbf{k}\left(\left(\mu_{n}, x_{n}\right),\left(\mu_{n}, x_{n}\right)\right)\left(Y^{2}-\mu_{n}^{2}\right) \\
= & \frac{1}{2}\left\|\sum_{n=1}^{N}\left(y_{n}-\mu_{n}\right) \Phi\left(\mu_{n}, x_{n}\right)\right\|_{\mathcal{H}}^{2}-\frac{1}{2} \sum_{n=1}^{N}\left(Y^{2}-\mu_{n}^{2}\right)\left\|\Phi\left(\mu_{n}, x_{n}\right)\right\|_{\mathcal{H}}^{2},
\end{aligned}
$$

which immediately implies (27). 


\section{Resolution}

This subsection makes the next step in our proof of Theorem 1 Our goal is to prove the following result (although we will need a slight modification of this result rather than the result itself).

Theorem 4 Let $\mathcal{F}$ be an $R K H S$ on $\mathbf{X}$ with reproducing kernel $\mathbf{k}$. The forecasts $\mu_{n} \in[-Y, Y]$ output by the ALN with parameter $\mathbf{k}$ always satisfy

$$
\left|\sum_{n=1}^{N}\left(y_{n}-\mu_{n}\right) D\left(x_{n}\right)\right| \leq Y \mathbf{c}_{\mathcal{F}}\|D\|_{\mathcal{F}} \sqrt{N}
$$

for all $N$ and all functions $D \in \mathcal{F}$.

Proof Using (27) with $\Phi$ being the feature mapping $x \in \mathbf{X} \mapsto \mathbf{k}_{x} \in \mathcal{F}$, we obtain

$$
\begin{aligned}
&\left|\sum_{n=1}^{N}\left(y_{n}-\mu_{n}\right) D\left(x_{n}\right)\right|=\left|\sum_{n=1}^{N}\left(y_{n}-\mu_{n}\right)\left\langle\mathbf{k}_{x_{n}}, D\right\rangle_{\mathcal{F}}\right| \\
&=\left|\left\langle\sum_{n=1}^{N}\left(y_{n}-\mu_{n}\right) \mathbf{k}_{x_{n}}, D\right\rangle_{\mathcal{F}}\right| \leq\left\|\sum_{n=1}^{N}\left(y_{n}-\mu_{n}\right) \mathbf{k}_{x_{n}}\right\|\|D\|_{\mathcal{F}} \\
& \leq\|D\|_{\mathcal{F}} \sqrt{\sum_{n=1}^{N} Y^{2} \mathbf{k}\left(x_{n}, x_{n}\right)} \leq Y \mathbf{c}_{\mathcal{F}}\|D\|_{\mathcal{F}} \sqrt{N}
\end{aligned}
$$

for any $D \in \mathcal{F}$.

Theorem 4 can be interpreted as asserting that the ALN has a good "resolution" when $\mathcal{F}$ is a universal RKHS; for details, see [44].

\section{Mixing feature mappings}

In the proof of Theorem 1 we will mix the feature mapping $\Phi_{0}(\mu, x):=\mu$ (into $\left.\mathcal{H}_{0}:=\mathbb{R}\right)$ and the feature mapping $\Phi_{1}(\mu, x):=\mathbf{k}_{x}$ used in the proof of Theorem 廿4 (we will have to achieve two goals simultaneously). This can be done using the following corollary of Theorem 3

Corollary 4 Let $\Phi_{j}:[-Y, Y] \times \mathbf{X} \rightarrow \mathcal{H}_{j}, j=0,1$, be forecast-continuous mappings from $[-Y, Y] \times \mathbf{X}$ to Hilbert spaces $\mathcal{H}_{j}$. The forecasts $\mu_{n} \in[-Y, Y]$ output by the ALN with a suitable kernel parameter always satisfy

$$
\left\|\sum_{n=1}^{N}\left(y_{n}-\mu_{n}\right) \Phi_{j}\left(\mu_{n}, x_{n}\right)\right\|_{\mathcal{H}_{j}}^{2} \leq Y^{2} \sum_{n=1}^{N}\left(\left\|\Phi_{0}\left(\mu_{n}, x_{n}\right)\right\|_{\mathcal{H}_{0}}^{2}+\left\|\Phi_{1}\left(\mu_{n}, x_{n}\right)\right\|_{\mathcal{H}_{1}}^{2}\right)
$$

for all $N$ and for both $j=0$ and $j=1$. 
Proof Define the direct sum $\mathcal{H}$ of $\mathcal{H}_{0}$ and $\mathcal{H}_{1}$ as the Cartesian product $\mathcal{H}_{0} \times \mathcal{H}_{1}$ equipped with the inner product

$$
\left\langle g, g^{\prime}\right\rangle_{\mathcal{H}}=\left\langle\left(g_{0}, g_{1}\right),\left(g_{0}^{\prime}, g_{1}^{\prime}\right)\right\rangle_{\mathcal{H}}:=\sum_{j=0}^{1}\left\langle g_{j}, g_{j}^{\prime}\right\rangle_{\mathcal{H}_{j}} .
$$

Now we can define $\Phi:[-Y, Y] \times \mathbf{X} \rightarrow \mathcal{H}$ by

$$
\Phi(\mu, x):=\left(\Phi_{0}(\mu, x), \Phi_{1}(\mu, x)\right) ;
$$

the corresponding kernel is

$$
\begin{aligned}
\mathbf{k}\left((\mu, x),\left(\mu^{\prime}, x^{\prime}\right)\right):= & \left\langle\Phi(\mu, x), \Phi\left(\mu^{\prime}, x^{\prime}\right)\right\rangle_{\mathcal{H}} \\
& =\sum_{j=0}^{1}\left\langle\Phi_{j}(\mu, x), \Phi_{j}\left(\mu^{\prime}, x^{\prime}\right)\right\rangle_{\mathcal{H}_{j}}=\sum_{j=0}^{1} \mathbf{k}_{j}\left((\mu, x),\left(\mu^{\prime}, x^{\prime}\right)\right),
\end{aligned}
$$

where $\mathbf{k}_{0}$ and $\mathbf{k}_{1}$ are the kernels corresponding to $\Phi_{0}$ and $\Phi_{1}$, respectively. It is clear that this kernel is forecast-continuous. Applying the ALN to it and using [27), we obtain

$$
\begin{gathered}
\left\|\sum_{n=1}^{N}\left(y_{n}-\mu_{n}\right) \Phi_{j}\left(\mu_{n}, x_{n}\right)\right\|_{\mathcal{H}_{j}}^{2} \\
\leq\left\|\left(\sum_{n=1}^{N}\left(y_{n}-\mu_{n}\right) \Phi_{0}\left(\mu_{n}, x_{n}\right), \sum_{n=1}^{N}\left(y_{n}-\mu_{n}\right) \Phi_{1}\left(\mu_{n}, x_{n}\right)\right)\right\|_{\mathcal{H}}^{2} \\
=\left\|\sum_{n=1}^{N}\left(y_{n}-\mu_{n}\right) \Phi\left(\mu_{n}, x_{n}\right)\right\|_{\mathcal{H}}^{2} \leq Y^{2} \sum_{n=1}^{N}\left\|\Phi\left(\mu_{n}, x_{n}\right)\right\|_{\mathcal{H}}^{2} \\
=Y^{2} \sum_{n=1}^{N} \sum_{j=0}^{1}\left\|\Phi_{j}\left(\mu_{n}, x_{n}\right)\right\|_{\mathcal{H}_{j}}^{2} .
\end{gathered}
$$

Merging $\Phi_{0}(\mu, x)=\mu$ and $\Phi_{1}(\mu, x)=\mathbf{k}_{x}$ by Corollary 4 we obtain

$$
\begin{array}{r}
\left|\sum_{n=1}^{N}\left(y_{n}-\mu_{n}\right) \mu_{n}\right|=\left\|\sum_{n=1}^{N}\left(y_{n}-\mu_{n}\right) \Phi_{0}\left(\mu_{n}, x_{n}\right)\right\|_{\mathbb{R}} \\
\leq Y \sqrt{\sum_{n=1}^{N}\left(\mu_{n}^{2}+\mathbf{k}\left(x_{n}, x_{n}\right)\right)}
\end{array}
$$


and, using (29),

$$
\begin{array}{r}
\left|\sum_{n=1}^{N}\left(y_{n}-\mu_{n}\right) D\left(x_{n}\right)\right| \leq\left\|\sum_{n=1}^{N}\left(y_{n}-\mu_{n}\right) \mathbf{k}_{x_{n}}\right\|_{\mathcal{F}}\|D\|_{\mathcal{F}} \\
=\left\|\sum_{n=1}^{N}\left(y_{n}-\mu_{n}\right) \Phi_{1}\left(\mu_{n}, x_{n}\right)\right\|\left\|_{\mathcal{F}}\right\| D \|_{\mathcal{F}} \\
\leq Y\|D\|_{\mathcal{F}} \sqrt{\sum_{n=1}^{N}\left(\mu_{n}^{2}+\mathbf{k}\left(x_{n}, x_{n}\right)\right)},
\end{array}
$$

for each function $D \in \mathcal{F}$.

\section{Proof proper}

The proof is based on the elementary inequality

$$
\begin{aligned}
& \sum_{n=1}^{N}\left(y_{n}-\mu_{n}\right)^{2} \\
& =\sum_{n=1}^{N}\left(y_{n}-D\left(x_{n}\right)\right)^{2}+2 \sum_{n=1}^{N}\left(D\left(x_{n}\right)-\mu_{n}\right)\left(y_{n}-\mu_{n}\right)-\sum_{n=1}^{N}\left(D\left(x_{n}\right)-\mu_{n}\right)^{2} \\
& \leq \sum_{n=1}^{N}\left(y_{n}-D\left(x_{n}\right)\right)^{2}+2 \sum_{n=1}^{N}\left(D\left(x_{n}\right)-\mu_{n}\right)\left(y_{n}-\mu_{n}\right)
\end{aligned}
$$

(the intermediate equality follows from $\left.a^{2}=(a-b)^{2}+2 a b-b^{2}\right)$. Using this

inequality and (30)-31), we obtain for the $\mu_{n} \in[-Y, Y]$ output by the ALN with the merged kernel as parameter:

$$
\begin{aligned}
& \sum_{n=1}^{N}\left(y_{n}-\mu_{n}\right)^{2} \\
& \leq \sum_{n=1}^{N}\left(y_{n}-D\left(x_{n}\right)\right)^{2}+2\left|\sum_{n=1}^{N} \mu_{n}\left(y_{n}-\mu_{n}\right)\right|+2\left|\sum_{n=1}^{N} D\left(x_{n}\right)\left(y_{n}-\mu_{n}\right)\right| \\
& \leq \sum_{n=1}^{N}\left(y_{n}-D\left(x_{n}\right)\right)^{2}+2 Y\left(\|D\|_{\mathcal{F}}+1\right) \sqrt{\sum_{n=1}^{N}\left(\mu_{n}^{2}+\mathbf{k}\left(x_{n}, x_{n}\right)\right)} \\
& \leq \sum_{n=1}^{N}\left(y_{n}-D\left(x_{n}\right)\right)^{2}+2 Y\left(\|D\|_{\mathcal{F}}+1\right) \sqrt{Y^{2}+\mathbf{c}_{\mathcal{F}}^{2}} \sqrt{N} .
\end{aligned}
$$

\section{Proof of Theorem 2}

In this section we will modify (essentially, further simplify) the proof of Theorem पgiven in the previous section to obtain the proof of Theorem [2] 


\section{K29 algorithm}

A kernel $\mathbf{k}$ on $[-Y, Y] \times \mathbf{X}$ is K29-admissible if the function $\mathbf{k}\left((\mu, x),\left(\mu^{\prime}, x^{\prime}\right)\right)$ is continuous in $\mu \in[-Y, Y]$ for all fixed $\mu^{\prime} \in[-Y, Y], x \in \mathbf{X}$, and $x^{\prime} \in \mathbf{X}$. For such a kernel the function

$$
S_{n}(\mu):=\sum_{i=1}^{n-1} \mathbf{k}\left(\left(\mu, x_{n}\right),\left(\mu_{i}, x_{i}\right)\right)\left(y_{i}-\mu_{i}\right)
$$

is continuous in $\mu \in[-Y, Y]$.

The K29 ALGORITHM

Parameter: K29-admissible kernel $\mathbf{k}$ on $[-Y, Y] \times \mathbf{X}$

FOR $n=1,2, \ldots$ :

Read $x_{n} \in \mathbf{X}$.

Define $S_{n}:[-Y, Y] \rightarrow \mathbb{R}$ by (33).

Output any root $\mu \in[-Y, Y]$ of $S_{n}(\mu)=0$ as $\mu_{n}$;

if there are no roots, set $\mu_{n}:=Y \operatorname{sign} S_{n}$.

Read $y_{n} \in[-Y, Y]$.

END FOR.

Let us say that a feature mapping $\Phi(\mu, x)$ is K29-admissible if the kernel $\mathbf{k}$ defined by (26) is K29-admissible.

Theorem 5 Let $\mathbf{k}$ be the kernel defined by 26) for a K29-admissible feature mapping $\Phi:[-Y, Y] \times \mathbf{X} \rightarrow \mathcal{H}$. The K29 algorithm with parameter $\mathbf{k}$ outputs $\mu_{n} \in[-Y, Y]$ such that

$$
\left\|\sum_{n=1}^{N}\left(y_{n}-\mu_{n}\right) \Phi\left(\mu_{n}, x_{n}\right)\right\|_{\mathcal{H}}^{2} \leq \sum_{n=1}^{N}\left(y_{n}-\mu_{n}\right)^{2}\left\|\Phi\left(\mu_{n}, x_{n}\right)\right\|_{\mathcal{H}}^{2}
$$

always holds for all $N=1,2, \ldots$.

Proof Following the K29 algorithm Predictor ensures that Skeptic will never increase his capital with the strategy

$$
s_{n}:=\sum_{i=1}^{n-1} \mathbf{k}\left(\left(\mu_{n}, x_{n}\right),\left(\mu_{i}, x_{i}\right)\right)\left(y_{i}-\mu_{i}\right)
$$


which implies

$$
\begin{aligned}
0 \geq & \mathcal{K}_{N}-\mathcal{K}_{0}=\sum_{n=1}^{N} s_{n}\left(y_{n}-\mu_{n}\right) \\
= & \sum_{n=1}^{N} \sum_{i=1}^{n-1} \mathbf{k}\left(\left(\mu_{n}, x_{n}\right),\left(\mu_{i}, x_{i}\right)\right)\left(y_{n}-\mu_{n}\right)\left(y_{i}-\mu_{i}\right) \\
= & \frac{1}{2} \sum_{n=1}^{N} \sum_{i=1}^{N} \mathbf{k}\left(\left(\mu_{n}, x_{n}\right),\left(\mu_{i}, x_{i}\right)\right)\left(y_{n}-\mu_{n}\right)\left(y_{i}-\mu_{i}\right) \\
& -\frac{1}{2} \sum_{n=1}^{N} \mathbf{k}\left(\left(\mu_{n}, x_{n}\right),\left(\mu_{n}, x_{n}\right)\right)\left(y_{n}-\mu_{n}\right)^{2} \\
= & \frac{1}{2}\left\|\sum_{n=1}^{N}\left(y_{n}-\mu_{n}\right) \Phi\left(\mu_{n}, x_{n}\right)\right\|_{\mathcal{H}}^{2}-\frac{1}{2} \sum_{n=1}^{N}\left(y_{n}-\mu_{n}\right)^{2}\left\|\Phi\left(\mu_{n}, x_{n}\right)\right\|_{\mathcal{H}}^{2},
\end{aligned}
$$

which in turn implies (34).

\section{Mixing feature mappings}

Now we have the following corollary of Theorem 5

Corollary $\mathbf{5}$ Let $\Phi_{j}:[-Y, Y] \times \mathbf{X} \rightarrow \mathcal{H}_{j}, j=0,1$, be forecast-continuous mappings from $[-Y, Y] \times \mathbf{X}$ to Hilbert spaces $\mathcal{H}_{j}$. The forecasts $\mu_{n} \in[-Y, Y]$ output by the K29 algorithm with a suitable kernel parameter always satisfy

$$
\begin{aligned}
\| \sum_{n=1}^{N}\left(y_{n}-\mu_{n}\right) \Phi_{j}\left(\mu_{n}, x_{n}\right) & \|_{\mathcal{H}_{j}}^{2} \\
& \leq \sum_{n=1}^{N}\left(y_{n}-\mu_{n}\right)^{2}\left(\left\|\Phi_{0}\left(\mu_{n}, x_{n}\right)\right\|_{\mathcal{H}_{0}}^{2}+\left\|\Phi_{1}\left(\mu_{n}, x_{n}\right)\right\|_{\mathcal{H}_{1}}^{2}\right)
\end{aligned}
$$

for all $N$ and for both $j=0$ and $j=1$.

Proof Being forecast-continuous, the kernel $\mathbf{k}$ defined in the proof of Corollary 4 is a fortiori K29-admissible. Applying the K29 algorithm to it and using (34), we obtain

$$
\begin{aligned}
\left\|\sum_{n=1}^{N}\left(y_{n}-\mu_{n}\right) \Phi_{j}\left(\mu_{n}, x_{n}\right)\right\|_{\mathcal{H}_{j}}^{2} & \\
\leq\left\|\sum_{n=1}^{N}\left(y_{n}-\mu_{n}\right) \Phi\left(\mu_{n}, x_{n}\right)\right\|_{\mathcal{H}}^{2} & \leq \sum_{n=1}^{N}\left(y_{n}-\mu_{n}\right)^{2}\left\|\Phi\left(\mu_{n}, x_{n}\right)\right\|_{\mathcal{H}}^{2} \\
& =\sum_{n=1}^{N}\left(y_{n}-\mu_{n}\right)^{2} \sum_{j=0}^{1}\left\|\Phi_{j}\left(\mu_{n}, x_{n}\right)\right\|_{\mathcal{H}_{j}}^{2} .
\end{aligned}
$$


Merging $\Phi_{0}(\mu, x)=\mu$ and $\Phi_{1}(\mu, x)=\mathbf{k}_{x}$ by Corollary $\mathbf{5}$ we obtain

$$
\begin{aligned}
& \left|\sum_{n=1}^{N}\left(y_{n}-\mu_{n}\right) \mu_{n}\right|=\left\|\sum_{n=1}^{N}\left(y_{n}-\mu_{n}\right) \Phi_{0}\left(\mu_{n}, x_{n}\right)\right\|_{\mathbb{R}} \\
& \quad \leq \sqrt{\sum_{n=1}^{N}\left(y_{n}-\mu_{n}\right)^{2}\left(\mu_{n}^{2}+\mathbf{k}\left(x_{n}, x_{n}\right)\right)} \leq \sqrt{\mathbf{c}_{\mathcal{F}}^{2}+Y^{2}} \sqrt{\sum_{n=1}^{N}\left(y_{n}-\mu_{n}\right)^{2}}
\end{aligned}
$$

and, using (29),

$$
\begin{array}{r}
\left|\sum_{n=1}^{N}\left(y_{n}-\mu_{n}\right) D\left(x_{n}\right)\right| \leq\left\|\sum_{n=1}^{N}\left(y_{n}-\mu_{n}\right) \mathbf{k}_{x_{n}}\right\|_{\mathcal{F}}\|D\|_{\mathcal{F}} \\
=\left\|\sum_{n=1}^{N}\left(y_{n}-\mu_{n}\right) \Phi_{1}\left(\mu_{n}, x_{n}\right)\right\|_{\mathcal{F}}\|D\|_{\mathcal{F}} \\
\leq\|D\|_{\mathcal{F}} \sqrt{\sum_{n=1}^{N}\left(y_{n}-\mu_{n}\right)^{2}\left(\mu_{n}^{2}+\mathbf{k}\left(x_{n}, x_{n}\right)\right)} \\
\leq\|D\|_{\mathcal{F}} \sqrt{\mathbf{c}_{\mathcal{F}}^{2}+Y^{2}} \sqrt{\sum_{n=1}^{N}\left(y_{n}-\mu_{n}\right)^{2}},
\end{array}
$$

for each function $D \in \mathcal{F}$.

\section{Proof proper}

Using (32) and (35)-(36), we obtain for the $\mu_{n}$ output by the K29 algorithm with the merged kernel as parameter:

$$
\begin{aligned}
& \sum_{n=1}^{N}\left(y_{n}-\mu_{n}\right)^{2} \\
& \leq \sum_{n=1}^{N}\left(y_{n}-D\left(x_{n}\right)\right)^{2}+2\left|\sum_{n=1}^{N} \mu_{n}\left(y_{n}-\mu_{n}\right)\right|+2\left|\sum_{n=1}^{N} D\left(x_{n}\right)\left(y_{n}-\mu_{n}\right)\right| \\
& \leq \sum_{n=1}^{N}\left(y_{n}-D\left(x_{n}\right)^{2}+2 \sqrt{\mathbf{c}_{\mathcal{F}}^{2}+Y^{2}}\left(\|D\|_{\mathcal{F}}+1\right) \sqrt{\sum_{n=1}^{N}\left(y_{n}-\mu_{n}\right)^{2}} .\right.
\end{aligned}
$$

The inequality between the extreme terms of this chain is quadratic in

$$
\sqrt{\sum_{n=1}^{N}\left(y_{n}-\mu_{n}\right)^{2}}
$$


solving it, we obtain

$$
\begin{aligned}
& \sqrt{\sum_{n=1}^{N}\left(y_{n}-\mu_{n}\right)^{2}} \leq \sqrt{\sum_{n=1}^{N}\left(y_{n}-D\left(x_{n}\right)\right)^{2}+\left(\mathbf{c}_{\mathcal{F}}^{2}+Y^{2}\right)\left(\|D\|_{\mathcal{F}}+1\right)^{2}} \\
& +\sqrt{\mathbf{c}_{\mathcal{F}}^{2}+Y^{2}}\left(\|D\|_{\mathcal{F}}+1\right),
\end{aligned}
$$

which is equivalent to (4).

\section{The algorithms}

In this short section we extract the prediction strategy achieving (3) and (4) from our proof of Theorems 1 and 2 Replacing in (25) the kernel $\mathbf{k}\left((\mu, x),\left(\mu^{\prime}, x^{\prime}\right)\right)$ by the merged kernel $\mu \mu^{\prime}+\left\langle\mathbf{k}_{x}, \mathbf{k}_{x^{\prime}}\right\rangle_{\mathcal{F}}$, we obtain

$$
S_{n}(\mu)=\sum_{i=1}^{n-1}\left(\mu \mu_{i}+\mathbf{k}\left(x_{n}, x_{i}\right)\right)\left(y_{i}-\mu_{i}\right)-\left(\mu^{2}+\mathbf{k}\left(x_{n}, x_{n}\right)\right) \mu ;
$$

this immediately leads to the following explicit description for the prediction algorithm we used in the proof of Theorem [1

AN ALGORITHM ACHIEVING (3)

Parameter: the reproducing kernel $\mathbf{k}$ of $\mathcal{F}$

FOR $n=1,2, \ldots$ :

Read $x_{n} \in \mathbf{X}$.

Define $S_{n}(\mu)$ by (37) for all $\mu \in[-Y, Y]$.

Define $\mu_{n}$ as any root $\mu \in[-Y, Y]$ of $S_{n}(\mu)=0$;

if there are no roots, set $\mu_{n}:=Y \operatorname{sign} S_{n}$.

Read $y_{n} \in[-Y, Y]$.

END FOR.

To obtain an algorithm achieving (4), it suffices to replace (37) by

$$
S_{n}(\mu)=\sum_{i=1}^{n-1}\left(\mu \mu_{i}+\mathbf{k}\left(x_{n}, x_{i}\right)\right)\left(y_{i}-\mu_{i}\right) .
$$

\section{Acknowledgments}

I am grateful to Nicolò Cesa-Bianchi and Alex Smola for useful remarks. This work was partially supported by MRC (grant S505/65) and the Royal Society. 


\section{References}

[1] Robert A. Adams and John J. F. Fournier. Sobolev Spaces, volume 140 of Pure and Applied Mathematics. Academic Press, Amsterdam, second edition, 2003.

[2] Nachman Aronszajn. La théorie générale des noyaux reproduisants et ses applications, première partie. Proceedings of the Cambridge Philosophical Society, 39:133-153 (additional note: p. 205), 1944. The second part of this paper is 3 .

[3] Nachman Aronszajn. Theory of reproducing kernels. Transactions of the American Mathematical Society, 68:337-404, 1950.

[4] Marc Atteia. Hilbertian Kernels and Spline Functions, volume 4 of Studies in Computational Mathematics. Noth-Holland, Amsterdam, 1992.

[5] Katy S. Azoury and Manfred K. Warmuth. Relative loss bounds for on-line density estimation with the exponential family of distributions. Machine Learning, 43:211-246, 2001.

[6] Alain Berlinet and Christine Thomas-Agnan. Reproducing Kernel Hilbert Spaces in Probability and Statistics. Kluwer, Boston, 2004.

[7] Avrim Blum, Adam Kalai, and John Langford. Beating the hold-out: Bounds for $k$-fold and progressive cross-validation. In Proceedings of the Twelfth Annual Conference on Computational Learning Theory, pages 203208, New York, 1999. Association for Computing Machinery.

[8] Nicolò Cesa-Bianchi, Alex Conconi, and Claudio Gentile. A second-order perceptron algorithm. In Jyrki Kivinen and Robert H. Sloan, editors, Proceedings of the Fifteenth Annual Conference on Computational Learning Theory, volume 2375 of Lecture Notes in Artificial Intelligence, pages 121137. Springer, 2002.

[9] Nicolò Cesa-Bianchi, Alex Conconi, and Claudio Gentile. On the generalization ability of on-line learning algorithms. IEEE Transactions on Information Theory, 50:2050-2057, 2004.

[10] Nicolò Cesa-Bianchi, Philip M. Long, and Manfred K. Warmuth. Worstcase quadratic loss bounds for on-line prediction of linear functions by gradient descent. IEEE Transactions on Neural Networks, 7:604-619, 1996.

[11] Peter Craven and Grace Wahba. Smoothing noisy data with spline functions. Numerische Mathematik, 31:377-403, 1979.

[12] Felipe Cucker and Steve Smale. On the mathematical foundations of learning. Bulletin (New Series) of the American Mathematical Society, 39:1-49, 2002. 
[13] Luc Devroye, László Györfi, and Gábor Lugosi. A Probabilistic Theory of Pattern Recognition. Springer, New York, 1996.

[14] André Elisseeff and Olivier Bousquet. Stability and generalization. Journal of Machine Learning Research, 2:499-526, 2002.

[15] Jürgen Forster. On relative loss bounds in generalized linear regression. In Gabriel Ciobanu and Gheorghe Paun, editors, Proceedings of the Twelfth International Symposium on Fundamentals of Computation Theory, volume 1684 of Lecture Notes in Computer Science, pages 269-280, Berlin, 1999. Springer.

[16] Dean P. Foster. Prediction in the worst case. Annals of Statistics, 19:10841090, 1991.

[17] Dean P. Foster and Rakesh V. Vohra. Asymptotic calibration. Biometrika, 85:379-390, 1998.

[18] Alex Gammerman, Yuri Kalnishkan, and Vladimir Vovk. On-line prediction with kernels and the Complexity Approximation Principle. In Max Chickering and Joseph Halpern, editors, Proceedings of the Twentieth Annual Conference on Uncertainty in Artificial Intelligence, pages 170-176, Arlington, VA, 2004. AUAI Press.

[19] Chong Gu. Smoothing Spline ANOVA Models. Springer Series in Statistics. Springer, New York, 2002.

[20] G. H. Hardy, John E. Littlewood, and George Pólya. Inequalities. Cambridge University Press, Cambridge, second edition, 1952.

[21] Wassily Hoeffding. Probability inequalities for sums of bounded random variables. Journal of the American Statistical Association, 58:13-30, 1963.

[22] Sham M. Kakade and Dean P. Foster. Deterministic calibration and Nash equilibrium. In John Shawe-Taylor and Yoram Singer, editors, Proceedings of the Seventeenth Annual Conference on Learning Theory, volume 3120 of Lecture Notes in Computer Science, pages 33-48, Heidelberg, 2004. Springer.

[23] Sham M. Kakade, Matthias W. Seeger, and Dean P. Foster. Worst-case bounds for Gaussian process models. Working paper, http://www.cis. upenn. edu/ $\sim$ skakade/, accessed in November, 2005. To appear in Advances in Neural Information Processing Systems.

[24] Don Kimber and Philip M. Long. On-line learning of smooth functions of a single variable. Theoretical Computer Science, 148:141-156, 1995.

[25] Jyrki Kivinen and Manfred K. Warmuth. Exponential Gradient versus Gradient Descent for linear predictors. Information and Computation, 132:163, 1997. 
[26] Wee Sun Lee, Peter L. Bartlett, and Robert C. Williamson. The importance of convexity in learning with squared loss. IEEE Transactions on Information Theory, 44:1974-1980, 1998.

[27] Ehud Lehrer. Any inspection is manipulable. Econometrica, 69:1333-1347, 2001.

[28] Nick Littlestone. From on-line to batch learning. In Ronald Rivest, David Haussler, and Manfred K. Warmuth, editors, Proceedings of the Second Annual Workshop on Computational Learning Theory, pages 269-284, San Mateo, CA, 1989. Morgan Kaufmann.

[29] Philip M. Long. Improved bounds about on-line learning of smooth functions of a single variable. Theoretical Computer Science, 241:25-35, 2000.

[30] Herbert Meschkowski. Hilbertsche Räume mit Kernfunktion. Springer, Berlin, 1962.

[31] Sayan Mukherjee, Ryan Rifkin, and Tomaso Poggio. Regression and classification with regularization. In David D. Denison, Mark H. Hansen, Christopher C. Holmes, Bani K. Mallick, and Bin Yu, editors, Nonlinear Estimation and Classification, volume 171 of Lecture Notes in Statistics, pages 107-124. Springer, New York, 2003.

[32] Alvaro Sandroni. The reproducible properties of correct forecasts. International Journal of Game Theory, 32:151-159, 2003.

[33] Alvaro Sandroni, Rann Smorodinsky, and Rakesh V. Vohra. Calibration with many checking rules. Mathematics of Operations Research, 28:141153, 2003.

[34] Bernhard Schölkopf and Alexander J. Smola. Learning with Kernels. MIT Press, Cambridge, MA, 2002.

[35] Glenn Shafer and Vladimir Vovk. Probability and Finance: It's Only a Game! Wiley, New York, 2001.

[36] John Shawe-Taylor and Nello Cristianini. Kernel Methods for Pattern Analysis. Cambridge University Press, Cambridge, 2004.

[37] Steve Smale and Ding-Xuan Zhou. Shannon sampling II: Connections to learning theory. Applied and Computational Harmonic Analysis, 2005. In press.

[38] Alex J. Smola, Bernhard Schölkopf, and Klaus-Robert Müller. The connection between regularization operators and support vector kernels. Neural Networks, 11:637-649, 1998.

[39] Ingo Steinwart. On the influence of the kernel on the consistency of support vector machines. Journal of Machine Learning Research, 2:67-93, 2001. 
[40] Christine Thomas-Agnan. Computing a family of reproducing kernels for statistical applications. Numerical Algorithms, 13:21-32, 1996.

[41] Vladimir N. Vapnik. Statistical Learning Theory. Wiley, New York, 1998.

[42] Vladimir Vovk. Competitive on-line statistics. International Statistical Review, 69:213-248, 2001.

[43] Vladimir Vovk. Competitive on-line learning with a convex loss function. Technical Report arXiv:cs.LG/0506041 (version 3), arXiv.org e-Print archive, September 2005.

[44] Vladimir Vovk. Non-asymptotic calibration and resolution. Technical Report arXiv:cs. LG/0506004 (version 3), arXiv . org e-Print archive, August 2005.

[45] Vladimir Vovk and Glenn Shafer. Good randomized sequential probability forecasting is always possible, The Game-Theoretic Probability and Finance project, http://probabilityandfinance.com, Working Paper \#7, June 2003 (revised September 2004). To appear in Journal of the Royal Statistical Society B.

[46] Vladimir Vovk, Akimichi Takemura, and Glenn Shafer. Defensive forecasting. Technical Report arXiv:cs.LG/0505083, arXiv .org e-Print archive, May 2005.

[47] Grace Wahba. Spline Models for Observational Data, volume 59 of CBMSNSF Regional Conference Series in Applied Mathematics. SIAM, Philadelphia, PA, 1990.

[48] Robert C. Williamson, Alex Smola, and Bernhard Schölkopf. Generalization performance of regularization networks and support vector machines via entropy numbers of compact operators. IEEE Transactions on Information Theory, 47:2516-2532, 2001.

[49] Tong Zhang. Leave-one-out bounds for kernel methods. Neural Computation, 15:1397-1437, 2003. 